\section{EDUCATION}

Research, Innovation and Solutions on-line ${ }^{\circledR}$
PSYCHOLOGY

I+D+i
Electronic Journal of Research

in Educational Psychology



Editorial EOS

\title{
Percepción sobre el coste de pensar críticamente en universitarios chilenos y españoles
}

\author{
Jorge Valenzuela ${ }^{1,2}$, Carlos Saiz ${ }^{2}$ \\ ${ }^{1}$ Faculté de Psychologie et des Scences de l'Education, \\ Université Catholique de Louvain \\ ${ }^{2}$ Departamento de Psicología Básica, Universidad de Salamanca
}

\section{Bélgica / España}

Correspondencia: Jorge Valenzuela. Place du cardinal Mercier 10 1348, Louvain la Neuve. Bélgica. E-mail: valenzu@gmail.com

(C) Education \& Psychology I+D+i and Editorial EOS (Spain) 


\section{Resumen}

Introducción. El objetivo de esta comunicación es describir y comparar la percepción que tienen estudiantes de universidades públicas de Chile y España sobre el coste de utilizar el pensamiento crítico (Estudio 1). En este marco, interesa contrastar la hipótesis de que pensar críticamente es una actividad percibida como costosa, y que por lo tanto, se puede activar como tarea a través de procesos motivacionales. Y, por otra parte, evaluar el cambio en la percepción de este coste cuando los alumnos son sometidos a un entrenamiento en habilidades de pensamiento crítico (Estudio 2).

Método. En este estudio participaron un total de 429 estudiantes chilenos (169) y españoles (260) de ambos sexos (84,9\% mujeres) que fueron evaluados sobre la percepción del coste de pensar críticamente en términos de esfuerzo, concentración, tiempo y energía, a través de una escala tipo Lickert válida y fiable.

Resultados. Los resultados revelan en todas las submuestras que el pensamiento crítico es percibido como una actividad costosa, y no existen diferencias significativas entre los estudiantes chilenos y los españoles. Asimismo, se constata que, tras la instrucción, la percepción de coste se incrementa significativamente.

Discusión y Conclusiones. Los resultados apoyan la tesis de que los factores motivacionales deberían ser decisivos en la activación de recursos cognitivos y en el desempeño de habilidades del pensamiento. Por otra parte, el incremento en la percepción de coste podría explicarse por el feedback sobre la manera de pensar percibida en el contexto de las tareas de pensamiento crítico, y la explicitación de procedimientos adecuados de razonamiento durante la intervención instruccional.

Palabras Clave: Pensamiento crítico; percepción de costo; motivación; universitarios; esfuerzo, concentración, tiempo y energía.

Recibido: 20/01/10 Aceptación Inicial: 20/01/10 Aceptación Definitiva: 09/04/10 


\title{
How Chilean and Spanish university students perceive the cost of thinking critically
}

\begin{abstract}
Introduction. Our aim on this communication is to describe and compare the perception that Chilean and Spanish public university students have about the cost of critical thinking use (Study 1). In this scope, we have tested the hypothesis that thinking critically it is an activity perceived as costly and therefore it is activated as a task through motivational processes. In a second study, we evaluated the student perception change of this cost under a specific pedagogical intervention in critical thinking abilities (Study 2).
\end{abstract}

Method. In these studies 429 university students of both sexes (84,9\% females) from Chile (169) and Spain (260) participated. They were evaluated on their perception of the cost of thinking critically in terms of effort, concentration, time and energy by means of a Lickert scale.

Results. In the first study, the results reveal in all of the sub-samples that critical thinking is perceived as a costly activity, with no significant differences between Chilean and Spanish students. Moreover, in the second study we confirm that after the instruction, the cost perception is increased significantly.

Discussion and Conclusion. The results support the thesis that motivational factors would be important for cognitive resources activation and the performances on thinking abilities. On the other hand, the increase in the cost perception could be explained by feedback on its way of thinking received in a critical thinking tasks context, and the clarification to the suitable process of reasoning during this pedagogical intervention.

Keywords: Critical Thinking; cost perception; motivation; university students; effort, concentration, time, energy.

Received: 01/20/10 Initial Acceptance: 01/20/10 Definitive Acceptance: 04/09/10 


\section{Introducción}

El pensamiento crítico es pensamiento razonado y reflexivo que se centra en que la persona pueda decidir qué creer o qué hacer (Ennis, 1989). Es la clase de pensamiento que está implicado en resolver problemas, en formular inferencias, en calcular probabilidades y en tomar decisiones (Halpern, 2006). Es un pensamiento de orden superior, y como tal, no es automático (Mertes, 1991), sino que requiere, autodeterminación, reflexión, esfuerzo, autocontrol y por otra parte, la utilización de estrategias metacognitivas (Sungur, 2007; Ugartetxea, 2002), puesto que durante su ejecución no se evalúa solamente el resultado sino también el proceso mismo que ha llevado a ese resultado (Evans, 2003; Noveck, Mercier, Rossi, y Van der Henst, 2007; Phan, 2006). En otros términos, pensar críticamente implica tomar la decisión de activar una serie de recursos cognitivos para poner en marcha y mantener la ejecución y supervisión de una serie de reglas y procedimientos que nos permiten razonar correctamente, solucionar problemas y tomar decisiones (Saiz, 2002; Saiz y Rivas, 2008).

La gran mayoría de los teóricos del campo concuerdan en que existirían dos componentes principales en el pensamiento crítico. Por una parte, las habilidades asociadas al razonamiento, solución de problemas y toma de decisiones (Saiz, 2002), y por otra, el componente motivacional que activaría dichas habilidades. Tradicionalmente se ha tendido a abordar esta segunda dimensión desde la perspectiva de las disposiciones, constructo con un fuerte influencia de la filosofía (Facione, 1990; Tishman y Andrade, 2004). Sin embargo, parece ser que analizar esta dimensión a partir de una perspectiva alternativa, como el marco teórico de la motivación, puede ser de más beneficioso tanto en términos teóricos como prácticos (Valenzuela y Nieto, 2008b, 2009) En primer lugar porque ofrece más pistas para la intervención con vistas a una mejora de estas capacidades, y por otro tiende a tener una mejor capacidad predictiva (Valenzuela y Nieto, 2009).

Nuestra opción ha sido considerar la motivación desde la perspectiva del modelo expectancy and value (Eccles et al., 1983; Eccles y Wigfield, 2002; Wigfield y Eccles, 1992; 2002; Wigfield, Eccles y Roeser, 1998) Esta mirada centra la atención en la motivación de logro en una tarea, en este caso, de carácter cognitiva. En esta perspectiva, la motivación por pensar críticamente depende de las expectativas (expectancy) que tiene la persona de realizar la tarea de manera apropiada, constructo que en la práctica sería equivalente al de autoeficacia de Bandura (1977; Bandura, Barbaranelli, Caprara, y Pastorelli, 1996; Eccles, et al., 1983; 
Pajares, 1996; Wigfield, et al., 1998) y al valor (value) que la persona asigna a esa tarea. Dicho valor se compone, a su vez, del grado de importancia, utilidad e interés que suscita la tarea y por el coste que se está dispuesto a asumir para llevarla a cabo (Eccles, et al., 1983; Neuville, Bourgeois, y Frenay, 2004; Wigfield y Eccles, 1992).

La actividad de pensar críticamente es costosa en términos cognitivos (Evans, 2003) e implica una dosis variable de esfuerzo, concentración, energía y tiempo, y, aunque asumimos que las personas percibirían la actividad de pensar crítica o rigurosamente como una actividad costosa, no tenemos evidencia empírica global de en qué magnitud esto es así. Como sucede con una gran cantidad de actividades, el desempeño en el pensamiento crítico podría estar influido por la percepción del coste que para la persona tiene la utilización de este tipo de pensamiento. En este sentido, datos preliminares recogidos por nuestro equipo indican que la percepción del coste de utilizar el pensamiento crítico, es decir, la percepción de que pensar críticamente es costosa, que implica invertir tiempo, esfuerzo, energía, concentración, estaría asociado de manera negativa con el desempeño en las habilidades del pensamiento crítico ( $\mathrm{r}=$ $-.233, p<.011)$ y específicamente con las tareas de deducción $(\mathrm{r}=-.182, p<.048)$ e inducción $(\mathrm{r}=-.213, p<.021)$. Sin embargo, esta relación no sería directa sino que estaría mediada por la motivación y, más específicamente, por las expectativas de realizar adecuadamente la tarea de pensar críticamente.

Sabemos que la motivación en general y más específicamente las expectativas, como elemento de la motivación, están relacionadas con las creencias que tenemos sobre la tarea, entre ellas, la percepción de la dificultad de la misma (Eccles, et al., 1983; Eccles y Wigfield, 2002; Visdómine-Lozano y Luciano, 2006) y sabemos además que las expectativas se correlacionan positivamente con el desempeño en habilidades de pensamiento crítico (Valenzuela y Nieto, 2008a). Por lo anterior, sería lógico esperar que la percepción del coste que involucra pensar críticamente incida sobre las expectativas de realizar adecuadamente esta tarea, y que a través de ellas, actúe sobre el desempeño en pensamiento crítico.

Dado que no existen estudios puntuales sobre la percepción del coste de utilización de las habilidades de pensamiento crítico, hemos querido explorar un poco más a fondo la percepción que los universitarios tienen sobre el coste que para ellos implica el pensar de manera rigurosa. Asimismo, nos interesa identificar cuáles son los elementos que más pesan en esta percepción comparando estos resultados en dos muestras de universitarios españoles y chile- 
nos y por sexo. Por otra parte, nos parece importante contrastar también la tesis de que el coste de utilización del pensamiento crítico estaría asociado con las expectativas.

Adicionalmente, nos ha interesado examinar qué ocurre con el coste de utilización del pensamiento crítico cuando los alumnos se ven expuestos a una intervención de enseñanza explícita de habilidades de pensamiento crítico.

\section{Estudio 1: Percepción de Coste en la utilización del pensamiento crítico entre universita- rios chilenos y españoles}

En este estudio se describe el coste de utilización del pensamiento crítico en universitarios chilenos y españoles y se contrastan las percepciones sobre el coste que implica un pensamiento crítico y riguroso.

\section{Método}

\section{Participantes}

La muestra estuvo constituida por universitarios chilenos $(n=169)$ y españoles $(n=260)$ de ambos sexos (Chile 83,8\% mujeres; España 86\% mujeres) de las carreras de psicología y educación, pertenecientes a 2 instituciones universitarias chilenas y a otras 2 españolas, en un rango de edad entre 18 y 30 años, con una media de 21,06 años $(s d=2,1)$. En todos los casos, se trató de universidades públicas a las que se accede a través de un proceso de selectividad. La participación fue voluntaria. En ambos casos, la participación de los alumnos fue retribuidos. En el caso de los estudiantes españoles, recibieron una pequeña recompensa académica consistente en puntos en la asignatura de psicología del pensamiento, mientras que en el caso chileno, a los alumnos se les ofreció un certificado de participación como informante de la investigación, certificado opcional que la gran mayoría solicitó. 


\section{Instrumentos}

Para evaluar el coste de utilización de las habilidades intelectuales se utilizó una escala ad-hoc de 4 ítems en la cual se le pregunta a la persona cuánta concentración, esfuerzo, tiempo y energía requiere pensar de manera crítica y rigurosa. La escala tipo Lickert tenía valores que iban de 1 a 6 , y donde 1 significaba poco y 6 significaba mucho. Dado que el pilotaje de los ítems no evidenció problemas de comprensión de los términos, no hubo explicitación de la definición de cada uno de ellos.

La escala se estructura en torno a un solo factor en todas las sub muestras así como en la muestra total, $(\mathrm{KMO}=.754 ; 1$ factor, eigenvalue $=2,425 ; 60,665 \%$ de varianza $)$ y su nivel de confiabilidad ( $\alpha$ de Cronbach) varía, para las diferentes muestras, entre 688 y .794 (ver tabla 2) no encontrándose diferencias estadísticamente significativas entre ellas $[M(3)=$ $3,8043 p<.283]$. Para la comparación estadística se utilizó un análisis que contrasta estadísticamente alfas de Cronbach (Valenzuela, 2007) a partir de la fórmula de Hakstian y Whalen (1976). Cabe señalar además, que la escala no evidenció problemas de efecto techo, ni en la muestra general $[\mathrm{t}(447)=-48.267, p<.001]$, ni en ninguna de las submuestras.

Tabla 1. Estadísticos de fiabilidad

\begin{tabular}{lrcc}
\hline Universidad & $\mathrm{N}$ & Alfa de Cronbach N de elementos \\
\hline Chile & & & \\
Universidad chilena 1 & 37 & .793 & 4 \\
Universidad chilena 2 & 129 & .794 & 4 \\
España & & & \\
Universidad española 1 & 159 & .765 & 4 \\
Universidad española 2 & 101 & .688 & 4 \\
\hline Muestra total & 426 & .765 & 4 \\
\hline
\end{tabular}

Para evaluar diferentes medidas de motivación por el pensamiento crítico se utilizó la Escala Motivacional de Pensamiento Crítico EMPC (Valenzuela y Nieto, 2008b; Valenzuela, Nieto, y Saiz, 2009) la cual presenta una fiabilidad media de $\alpha=.763$, y específicamente en la subescala de expectativas una confiabilidad media de $\alpha=.720$. 


\section{Procedimiento}

Las escalas fueron administradas conjuntamente y de manera colectiva a través de un soporte informático on-line de encuestas, siendo contestada la prueba en su totalidad en un tiempo máximo de 8 minutos. Aunque la prueba no era anónima, dado que estos datos servirían para contrastarlos con una medida post, se aseguró a los participantes la estricta confidencialidad de los datos proporcionados.

\section{Resultados}

Percepción del coste de utilizar el pensamiento crítico en universitarios chilenos y españoles

Los resultados muestran que la media en la percepción del coste de utilizar el pensamiento crítico presenta niveles altos. En todas las submuestras, la media es significativamente superior al punto medio de la escala $[t(428)=32.529, p<.001]$. Resultados similares se encuentran cuando se analizan por separado las muestras chilenas $[t(168)=17.382, p<.001]$ y españolas $[t(259)=28.560, p<.001]$. Se observa que los participantes consideran costoso el pensar de manera crítica y rigurosa.

Un segundo aspecto a destacar es que las medias de todas las muestras son similares así como las desviaciones estándar, no encontrándose diferencias estadísticamente significativas entre los estudiantes chilenos y los españoles $[F(1,427)=1.726, p<.190]$. Tampoco se encontraron diferencias al contrastar las cuatro sub muestras $[F(3,427)=1.424, p<.235]$. Los participantes, españoles y chilenos, perciben de manera similar el coste de pensar críticamente.

Dentro de los elementos que componen la escala, la concentración aparece como el rasgo percibido cómo más relevante en relación con el coste de utilización del pensamiento crítico. De hecho, este rasgo presenta una puntuación significativamente superior a los tres aspectos evaluados tanto en las muestras chilenas $[F(3,167)=86.692 ; p<.001]$ como en las españolas $[F(3,259)=68.740, p<.001]$. Además, presenta la media más alta y tiene la menor variabilidad entre los cuatro aspectos evaluados que conforman el coste de utilización (ver 
Figura 1), lo que denota una alta convergencia en la percepción de utilizar el pensamiento crítico.

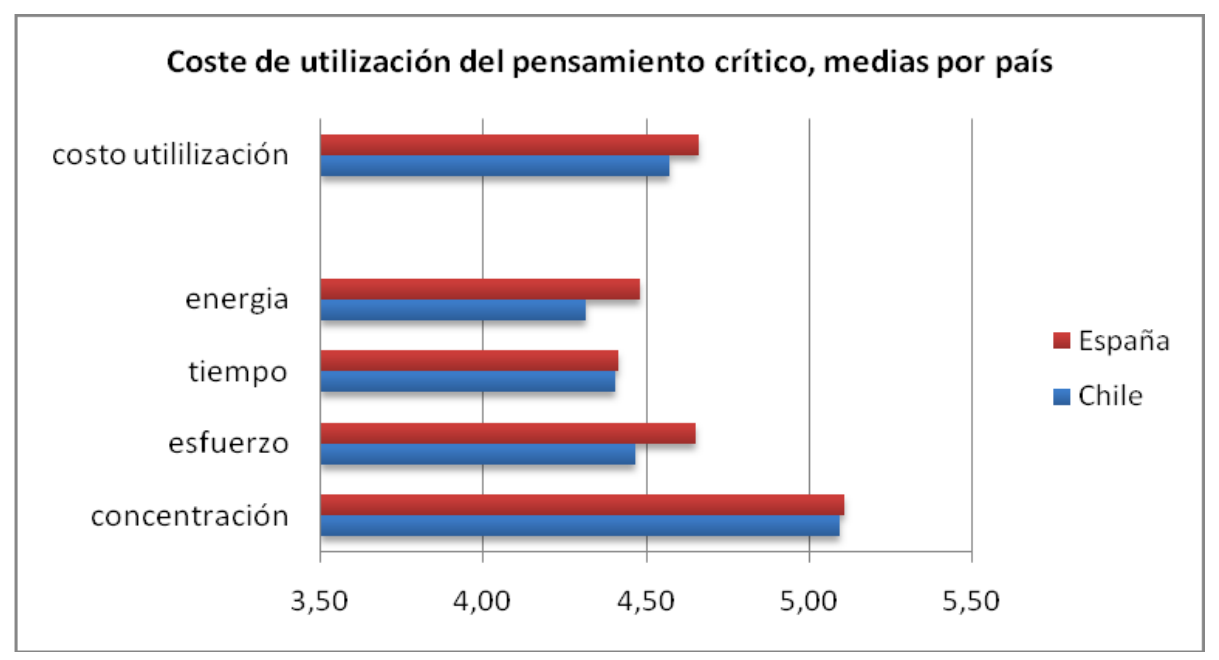

Figura 1 . Coste de Utilización del pensamiento crítico, medias por país

Tabla 2. Estadísticos descriptivos coste de utilización del pensamiento crítico por pais

\begin{tabular}{|c|c|c|c|c|c|}
\hline \multirow{2}{*}{$\frac{\text { País }}{\text { Chile }}$} & \multirow[b]{2}{*}{ concentración } & \multirow{2}{*}{$\begin{array}{ll}\mathrm{N} \quad \mathrm{M} \\
1692\end{array}$} & \multicolumn{2}{|c|}{ Min Max Mean } & \multirow{2}{*}{$\frac{\mathrm{SD}}{0.861}$} \\
\hline & & & 6 & 5.09 & \\
\hline & esfuerzo & 1691 & 6 & 4.47 & 1.080 \\
\hline & tiempo & 1692 & 6 & 4.40 & 0.959 \\
\hline & energia & 1692 & 6 & 4.31 & 1.151 \\
\hline & Coste utilizació & 1692 & 2.756 .00 & 4.5695 & 0.79991 \\
\hline \multicolumn{2}{|c|}{ España concentración } & 2603 & 6 & 5.11 & 0.711 \\
\hline & esfuerzo & 2602 & 6 & 4.65 & 0.894 \\
\hline & tiempo & 2602 & 6 & 4.41 & 0.822 \\
\hline & energia & 2601 & 6 & 4.48 & 1.045 \\
\hline & \multicolumn{5}{|c|}{ Coste utilización 2602.756 .004 .66250 .65634} \\
\hline
\end{tabular}

Al comparar el nivel de coste percibido según la variable sexo, y considerando la muestra general en su conjunto, encontramos que las mujeres perciben como más costosa la actividad de pensar críticamente $[F(1,338)=6.994 ; p<.009]$ que los hombres. En todos los casos se observa homoesticidad de la varianza entre ambos grupos.

Al realizar el análisis por país encontramos patrones diferentes en ambas muestras (ver Figura 2). En la muestra chilena no hay diferencias estadísticamente significativas entre hombres y mujeres $[F(1,143)=.504, p<.479]$ siendo homogéneas las varianzas en ambos 
grupos [Levene $(142)=.560, p<.455]$. En cambio, en la muestra de estudiantes españoles encontramos que las mujeres tienen una percepción de coste significativamente superior a la de sus pares varones $[F(1,228)=4.994, p<.001]$. En este caso, como en el caso chileno, la varianza en los grupos de hombres y mujeres son similares [Levene $(228)=.547, p<460]$.

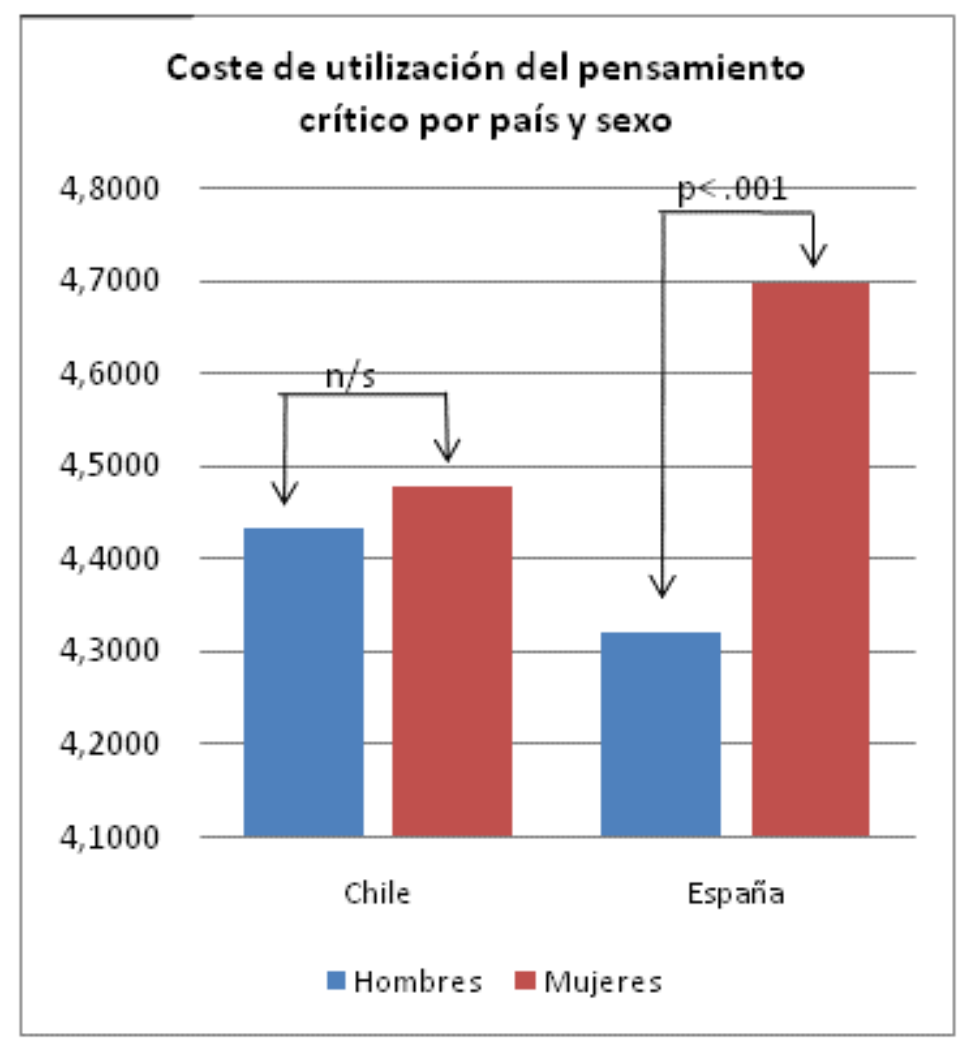

Figura 2. Coste de utilización del pensamiento crítico por país y sexo

Coste de utilización del pensamiento crítico y motivación: expectativas y valor de la tarea

Dado que la motivación, y muy especialmente las expectativas, dependen de la percepción que la persona tiene de la tarea, nos propusimos evaluar la hipótesis de que el coste de utilizar el pensamiento crítico estaría asociado significativamente y de manera negativa con las expectativas de desempeñar adecuadamente esta actividad.

Los resultados del análisis de correlación entre el coste de utilización del pensamiento crítico y las expectativas muestran patrones diferentes en las muestras chilenas y españolas. En la primera, estas dos variables correlacionan de manera significativa $(\mathrm{r}=.209 ; p<.009)$ mientras que sus pares españoles evidencian una correlación negativa y significativa entre ambas variables $(r=-164 ; p<.008)$. 
Además se constata que los elementos del coste de utilización que más inciden sobre las expectativas son, en el caso chileno el coste en términos de esfuerzo $[\beta=.206 ; t(168)=$ 2.727; $p$ <.007]; y en el caso de la muestra española, el coste en términos de energía [ $\beta=-.136$; $t(258)=-2.204, p<.028]$.

Por su parte, otro componente de la motivación, el valor, correlaciona con el coste de utilización de manera positiva y con valores muy similares en ambas muestras $(r=.235 ; p$ $<002$ y $r=209 ; p<.001$, en la muestra chilena y española respectivamente). Además de considerar el componente de valor de la tarea en su conjunto, es interesante llamar la atención sobre uno de los componentes: el coste (Neuville, et al., 2004; Neuville, Frenay, y Bourgeois, 2007). Se trata aquí del coste que se está dispuesto a asumir para realizar esa tarea y que no debemos confundir con la percepción de coste de utilizar el pensamiento crítico. A este respecto, encontramos que mientras en la muestra chilena no existe una correlación significativa entre coste de utilización del pensamiento crítico y el coste (componente del valor de la tarea) $(r=.027, p<.729)$, en el grupo de estudiantes españoles el coste percibido de pensar críticamente si se correlaciona significativamente con el coste que se está dispuesto a asumir para realizar dicha actividad $(r=.230, p<.001)$, a mayor coste en la realización de la tarea en términos de tiempo, concentración, esfuerzo y energía, mayor disposición para invertir en su realización. En este sentido, la percepción del coste de pensar de manera crítica es asumido en clave de reto, de desafío.

Tabla 3. Correlaciones Coste de Utlización y componentes de la motivación

\begin{tabular}{llcccccc}
\hline & & Expectativas & Valor & Importancia. & Utilidad & Coste & Interés \\
\hline Chile & Pearson r & $.212 * *$ & $.226^{* *}$ & $.295^{* *}$ & $.264^{* *}$ & .006 & $.167^{*}$ \\
& Sig. (2-tailed) & .006 & .003 & .000 & .001 & .938 & .030 \\
& $\mathrm{~N}$ & 169 & 169 & 169 & 169 & 169 & 169 \\
España & Pearson r & $\mathbf{- 1 6 4 * *}$ &. $\mathbf{2 0 9 * *}$ & $.140^{*}$ & $.158^{*}$ & $.230^{* *}$ & $.131^{*}$ \\
& Sig. (2-tailed) & .008 & .001 & .024 & .011 & .000 & .035 \\
& $\mathrm{~N}$ & 260 & 260 & 260 & 260 & 260 & 260 \\
\hline
\end{tabular}

*. Correlation significativa $p<.05$. **. Correlación significativa $p<.01$ 


\section{Estudio 2: Cambio en el Coste de utilización dentro de un contexto instruccional}

Un segundo estudio se lleva a cabo en el marco de una intervención de enseñanza de habilidades de pensamiento crítico. En él observamos los cambios producidos en la percepción de los alumnos sobre el coste de pensar críticamente al ser expuestos a una instrucción directa sobre habilidades de pensamiento (Saiz y Rivas, 2008).

\section{Participantes}

En este estudio participaron 94 alumnos (90.4\% mujeres) de cuarto año de la carrera de psicología en la fase de pre y post intervención. Los alumnos fueron retribuidos académicamente mediante la asignación de puntos en el curso de Psicología del pensamiento, en el marco del cual se realizó la instrucción de habilidades de pensamiento.

\section{Instrumentos}

Para evaluar el coste de utilización del pensamiento crítico se utilizó la escala ad-hoc anteriormente descrita.

\section{Procedimiento}

Los alumnos fueron evaluados antes y después de la intervención (diseño pre -post test), de manera colectiva y a través de soporte informático. La evaluación preinternvención tuvo lugar a mediados de septiembre, y la toma de datos del postintervención fue realizada durante la última semana de enero 2009, justo después de terminar el curso.

La intervención (Saiz, 2002; Saiz y Rivas, 2008), se enmarcó dentro de la asignatura de la asignatura de Psicología del pensamiento, de carácter obligatorio dentro del currículum de la carrera de psicología, duró 4 meses, y estuvo a cargo de uno de los investigadores. La formación consistió en la enseñanza directa de habilidades de pensamiento, organizadas en torno a las tres capacidades fundamentales del pensamiento crítico: razonamiento, solución de problemas y toma de decisiones (Saiz y Nieto, 2002). En relación a la primera, los alumnos recibieron instrucción directa sobre razonamiento deductivo (categórico y proposicional), razonamiento inductivo (generalizaciones inductivas, razonamiento causal, analógico e 
hipotético) y razonamiento práctico (habilidades de formalización evaluación y análisis de la argumentación). En relación a la segunda capacidad, los alumnos fueron instruidos en estrategias de resolución de problemas. Finalmente, en relación con la toma de decisiones, la instrucción se centró en el uso de heurísticos y probabilidades.

\section{Resultados}

La comparación preintervención/postintervención muestra un incremento significativo en la percepción de estos alumnos en torno al coste que implica el pensamiento crítico $[t(94)$ $=2.838, p<.006]$. No obstante, no todos los elementos implicados en el coste de utilización cambiaron significativamente. El coste en términos de tiempo $[t(93)=1.422, p<.158] \mathrm{y}$ energía $[t(93)=1.916, p<.058]$ no se modificaron de forma significativa. No obstante, la percepción del coste términos de esfuerzo $[t(93)=2.564, p<.012]$ que implica el pensamiento crítico se incrementó significativamente. De igual manera, ocurrió con el coste en términos de concentración $[t(93)=2.389, p<.019]$.

Al realizar los análisis según la variable sexo, aunque teniendo en consideración la baja representación del sexo masculino, es posible observar que entre los varones de la muestra no se observan cambios estadísticamente significativos ni en el índice de coste de utilización $[t(8)=-, 588, p<.573]$ ni en ninguno de los cuatro elementos que lo integran. Sin embargo, en la muestra femenina hubo un incremento significativo de su percepción del coste de utilizar el pensamiento crítico $[t(84)=3.223, p<.002]$ al igual que en todos los elementos que componen el índice, a excepción del coste en términos de tiempo [t(84) $=1.394, p<.167]$.

\section{Conclusiones}

Nuestro objetivo general en estos dos estudios complementarios era explorar la percepción que alumnos universitarios tienen sobre el coste de pensar críticamente. A partir de los resultados obtenidos en el primer estudio podemos sostener que pensar críticamente no sólo es costoso en términos cognitivos, sino que es percibido como tal, es decir que pensar críticamente es una actividad que implica una inversión importante de esfuerzo, concentración, energía y tiempo, y en ningún caso como algo que se hace habitualmente o de manera 
más o menos automática. Ello podría tener especial relevancia para el análisis motivacional en torno a esta actividad.

Esta percepción es similar en los grupos analizados. En ambas muestras la actividad de pensar críticamente es percibida de manera significativamente costosa y, por otra parte, el elemento que más incide en esta percepción es el coste en términos de concentración. Asimismo, llama la atención no encontrar diferencias intergrupo, cuando, por ejemplo, son cuestionados en torno a cuanto esfuerzo requiere pensar de manera crítica.

En relación con los análisis realizado según la variable sexo, constatamos que en general las mujeres perciben como más costoso el pensar críticamente. Sin embargo, estas diferencias observadas a nivel general son matizadas si consideramos el análisis por países. Mientras que en España, las mujeres efectivamente muestran una percepción de mayor coste al pensar críticamente que sus pares del sexo opuesto, en Chile no existen tales diferencias. Esto es consistente con las diferencias significativas $[F(1,334)=547.145, p<.001]$ que se observan entre las sub-muestras femeninas de ambos países en la subescala expectativas de la EMPC. Además, es importante señalar que la muestra española haya evidenciado diferencias significativas por sexo deba relacionarse con la percepción del coste de pensar de manera rigurosa está correlacionada con el coste que se está dispuesto a asumir para realizar adecuadamente la tarea. En otras palabras, estos resultados sugerirían que en la sub-muestra femenina española, si bien existe una percepción de que pensar de manera rigurosa es más costoso que para sus pares masculinos, este hecho es asumido en clave de reto o desafío, lo que podría ser aprovechado a la hora de diseñar intervenciones que apunten a la enseñanza de habilidades de pensamiento en este tipo de población.

Por otra parte, constatamos que el coste de utilización del pensamiento crítico se correlaciona con las expectativas de manera inversa en las muestras chilenas y española. Mientras que en la primera a una mayor percepción de coste se corresponde una mayor expectativa de realizar adecuadamente la tarea, en la muestra española sucede lo contrario. En la medida que aumenta la percepción de coste se observa una menor expectativa de que se podrá realizar bien la tarea. En el caso español, esta relación negativa entre expectativas y coste de utilización pudiera estar compensada, de alguna manera, por la asociación significativa entre la percepción de lo costoso que es pensar y el coste que se está dispuesto a asumir para realizar la tarea. En este sentido, los alumnos españoles, ante la dificultad de la tarea, pese a que creen 
que no obtendrán tan buenos resultados en la ejecución, están dispuestos a dejar otras cosas para invertir en pensar críticamente.

En relación al segundo estudio, el aumento significativo en la percepción del coste de pensar críticamente, podría explicarse considerando el conocimiento de la complejidad que implica un buen razonamiento a partir de la experiencia durante este curso. Este incremento, vendría a calibrar el grado de dificultad real que implica la tarea de pensar de manera "verdaderamente" rigurosa. Por otra parte, lo anterior es consistente con trabajos como los de Phan (2009) que muestran evidencia que correlaciona el esfuerzo con el pensamiento crítico ( $r=$ $.56, p<.01)$.

Una de las limitaciones de este trabajo es la composición de la muestra en términos de sexo, es decir, una muestra donde el sexo femenino está sobrerrepresentado. A pesar de ello, y de que la cantidad de sujetos en la muestra masculina sea muy pequeña, al realizar los contrastes se constata que, tanto en la muestra chilena como en la española, las varianzas entre hombres y mujeres no presentan diferencias significativas.

En síntesis, los resultados obtenidos al corroborar que el pensar críticamente y de manera rigurosa es efectivamente percibido como una actividad costosa, sugieren que las capacidades cognitivas deben ser, de alguna manera, activadas por un factor motivacional que evalúe si vale la pena el gasto cognitivo que implica, al menos en pensadores novatos que no han automatizado estas capacidades cognitivas. En este sentido, los resultados son más consistentes con la idea de que lo que activaría estas habilidades es un factor motivacional y no una disposición más o menos habitual de pensar de esta manera.

Como proyección de este trabajo creemos que es necesario un análisis más minucioso del efecto que eventualmente pueda tener el coste de utilización del pensamiento crítico. Es deseable pues, contar con una evaluación de habilidades de pensamiento crítico que recoja no sólo una estimación general, sino que pueda dar cuenta de manera más precisa de la forma en que la percepción sobre la dificultad de la tarea y del coste que ella implica, incide sobre el desempeño en tareas específicas del pensamiento crítico y a la vez, cómo esta percepción depende y se relaciona con variables metacognitivas que actúan como mediadoras del la regulación del esfuerzo (Sungur, 2007; Ugartetxea, 2002). 


\section{Referencias}

Bandura, A. (1977). Self-Efficacy: toward unifying theory of behavior change. Psychological Review, 84, 191-215.

Bandura, A., Barbaranelli, C., Caprara, G. V., y Pastorelli, C. (1996). Multifaceted impact of self-efficacy beliefs on academic functioning. Child Development, 67(3), 1206-1222.

Eccles, J., Adler, T. F., Futterman, R., Goff, S. B., Kaczala, C. M., Meece, J. L., et al. (1983). Expectancies, values, and academic behaviors Achievement and achievement motivation (pp. 75-146). San Francisco, CA: W.H.Freeman.

Eccles, J., y Wigfield, A. (2002). Motivational beliefs, Values and Goals. In S. T. Fiske, D. L. Schacter y C. Sahn-Waxler (Eds.), Annual Review of Psychology (pp. 109-132). Palo Alto, CA: Annual Reviews.

Ennis, R. H. (1989). Critical Thinking and Subject Specificity: Clarification and Needed Research. Educational Researcher, 18(3), 4-10.

Evans, J. S. B. T. (2003). In two minds: dual process accounts of reasoning. Trends in Cognitive Sciences, 7(10), 454-459.

Facione, P. (1990). APA Delphi Research Report, Critical Thinking: A Statement of Expert Consensus for Purposes of Educational Assessment and Instruction. ERIC Doc.No.: $E D, 315,423$.

Hakstian, R., y Whalen, T. (1976). A K-Sample Significance Test for Independent Alpha Coefficients. Psychometrika, 41(2), 219-231.

Halpern, D. (2006). Halpern Critical Thinking Assessment Using Everyday Situations: Background and scoring standards.Unpublished manuscript.

Mertes, L. (1991). Thinking and Writing. Middle School Journal, 22, 24-25.

Neuville, S., Bourgeois, É., y Frenay, M. (2004). The subjective task value: clarification of a construct. In S. Neuville (Ed.), La perception de la valeur des activités d'apprentissage : étude des déterminants et effets. Louvain la neuve: Unpublished Doctoral Thesis. Université Catholique de Louvain.

Neuville, S., Frenay, M., y Bourgeois, É. (2007). Task Value, Self-Efficacy and Goal Orientations: Impact on self-regulated learning, choice and performance among university students. Psychologica Belgica, 47(1), 95-117.

Noveck, I., Mercier, H., Rossi, S., y Van der Henst, J. B. (2007). Psichologie cognitive du raisonnement. In S. Rossi y J. B. Van der Henst (Eds.), Psychologies du raisonnement. Bruxelles: de Boeck. 
Pajares, F. (1996). Self-Efficacy beliefs in Academic Settings. Review of Educational Research, 66(4), 543-578.

Phan, H. (2006). Aproximación a los enfoques de aprendizaje, el pensamiento reflexivo, y las creencias epistemológicas: un enfoque de variables latentes. Electronic Journal of Research in Educational Psychology, 4(10), 577-610.

Phan, H. (2009). Reflective thinking, effort, persistence, disorganization, and academic performance: A mediational approach. Electronic Journal of Research in Educational Psychology, 7(3), 927-952.

Saiz, C. (Ed.). (2002). Pensamiento crítico: conceptos básicos y actividades prácticas. Madrid: Pirámide.

Saiz, C., y Nieto, A. (2002). Pensamiento crítico: capacidades y desarrollo. In C. Saiz (Ed.), Pensamiento crítico: conceptos básicos y actividades prácticas (pp. 15-19). Madrid: Pirámide.

Saiz, C., y Rivas, S. (2008). Intervenir para transferir en Pensamiento Crítico. Revista Praxis, 10(13), 129-149.

Sungur, S. (2007). Modeling the Relationships among Students' Motivational Beliefs, Metacognitive Strategy Use, and Effort Regulation. Scandinavian Journal of Educational Research, 51(3), 315-326.

Tishman, S., y Andrade, A. (2004). Disposiciones de pensamiento: Una revisión de teorías, prácticas y temas de actualidad.Unpublished manuscript.

Ugartetxea, J. (2002). La metacognición, el desarrollo de la autoeficacia y la motivación escolar. Revista de psicodidáctica, 13, 49-73.

Valenzuela, J. (2007). Alpha comparator (Version 2.0). Louvain la Neuve: Université Catholique de Louvain.

Valenzuela, J., y Nieto, A. (2008a). Motivación y desempeño en pensamiento crítico. . In J. A. González- Pienda y J. C. Núñez Pérez (Eds.), Psicología y Educación: Un lugar de encuentro (pp. 980-989). Oviedo: Ediciones Universidad de Oviedo.

Valenzuela, J., y Nieto, A. (2008b). Motivación y Pensamiento Crítico: Aportes para el estudio de esta relación. Revista Electrónica de Motivación y Emoción, 28(1), 1-8. Retrieved from http://reme.uji.es/reme/numero28/indexsp.html

Valenzuela, J., y Nieto, A. (2009). Motivación y Disposiciones como predictores del desempeño del Pensamiento Crítico. Paper presented at the VI Simposio de la Asociación de Motivación y Emoción (AME). Tenerife, Spain. 
Valenzuela, J., Nieto, A., y Saiz, C. (2009). Validation study of the Critical Thinking Motivational Scale. [Submited article].

Visdómine-Lozano, J., y Luciano, C. (2006). Locus de control y autorregulación conductual: revisiones conceptual y experimental. International Journal of Clinical and Health Psychology, 6(3), 729-751.

Wigfield, A., y Eccles, J. (1992). The development of achievement task values: A theoretical analysis. Developmental Review, 12, 265-310.

Wigfield, A., y Eccles, J. (2002). The development of competence beliefs, expectancies for success, and achievement values from childhood through adolescence Development of achievement motivation (pp. 91-120).

Wigfield, A., Eccles, J., y Roeser, R. W. (1998). Relations of young children's ability related beliefs to their subjective task values, performance, and effort. Paper presented at the "Motivation and affect in the classroom" (P. Pintrich, Organizer) at the International Congress of Applied Psychology. 